www.nature.com/ejhg

\title{
Allergic rhinitis - a total genome-scan for susceptibility genes suggests a locus on chromosome 4q24-q27
}

\author{
Annette Haagerup*,1, Torbjørn Bjerke ${ }^{2}$, Peter Oluf Schøitz ${ }^{3}$, Helle G Binderup ${ }^{1}$, \\ Ronald Dahl ${ }^{4}$ and Torben A Kruse ${ }^{\star, 5}$
}

\author{
${ }^{1}$ Institute of Human Genetics, Aarhus University, Aarhus, Denmark; ${ }^{2}$ ALK Abelló, Hørsholm, Denmark; \\ ${ }^{3}$ Department of Paediatrics, Aarhus University Hospital, Aarhus, Denmark; ${ }^{4}$ Department of Pneumology, Aarhus \\ University Hospital, Aarhus, Denmark; ${ }^{5}$ Department of Biochemistry and Clinical Genetics, Odense University \\ Hospital, Odense, Denmark
}

\begin{abstract}
Allergic rhinitis is a common disease of complex inheritance and is characterised by mucosal inflammation caused by allergen exposure. The genetics of closely related phenotypes such as asthma, atopy and to some extend atopic dermatitis has attracted attention in recent years. Genetic reports of allergic rhinitis on the contrary have as yet been most sparse. To identify candidate regions holding genes for allergic rhinitis we performed a total genome-scan on affected sib-pair families. From 100 Danish sib-pair families selected for allergy, families containing sib-pairs matching a phenotype definition of both clinical allergic rhinitis and confirmed specific allergy were chosen. Thirty-three affected sib-pair families qualified for the scan that was undertaken using $\mathbf{4 4 6}$ microsatellite markers. Non-parametric linkage results were obtained from MAPMAKER/SIBS computer program. The study revealed one major candidate region on chromosome 4q24q27 (LOD=2.83) and eight minor candidate regions 2q12-q33, 3q13, 4p15-q12, 5q13-q15, 6p24-p23, 12p13, $22 q 13$, and Xp21 (LOD=1.04-1.63) likely to contain susceptibility genes for allergic rhinitis. Our findings did not support a previous report of linkage of allergic rhinitis to chromosome 12q14-q24 but they added positive evidence to the asthma and atopy candidate regions $2 q 33$ and $6 \mathrm{p} 23$. Further identification of the specific genes involved in allergic rhinitis will give opportunities for improved diagnosis and treatment. European Journal of Human Genetics (2001) 9, 945-952.
\end{abstract}

Keywords: Rhinitis; genome-scan; locus; candidate region; allergy; genetics

\section{Introduction}

Rhinitis is a prevalent cause of personal morbidity and a significant contributor to costs of medical treatment. It is characterised by nasal itching and blockage, rhinorrhea and sneezing. Symptoms are in some definitions considered abnormal if lasting for at least $1 \mathrm{~h}$ a day on most days. ${ }^{1}$

*Correspondence: Annette Haagerup, Institute of Human Genetics, Aarhus University, Aarhus, Denmark. Tel: +45 8942 1680;

Fax: +45 8612 3173; E-mail: AH@humgen.au.dk or Professor TA Kruse, Department of Biochemistry and Clinical Genetics, Odense University Hospital, Odense, Denmark. E-mail: Torben.Kruse@ouh.fyns-amt.dk Received 10 July 2001; revised 26 October 2001; accepted 6 November 2001
Although sometimes treated with ease, rhinitis often significantly influences a patient's quality of life.

Allergic rhinitis is by far the most common form of rhinitis, affecting $10-30 \%$ of adults and up to $40 \%$ of children. ${ }^{2}$ Genetic factors account for $75 \%$ and environmental factors for $25 \%$ of disease risk. ${ }^{3}$ Allergic rhinitis often affects patients with other co-existing manifestations of allergy such as asthma and atopic dermatitis. Genetic studies of allergy have mainly reported results on asthma or general atopy phenotypes and only a few papers have evaluated allergic rhinitis.

The aim of this study was systematically to search the genome for candidate regions containing genes predisposing to allergic rhinitis. Therefore we undertook a comprehensive genome scan using 446 highly informative markers in 
thoroughly characterised Danish patient material. Thus, we present here results of the first genome-scan of the phenotype allergic rhinitis. Identification of genes predisposing to allergic rhinitis will not only improve knowledge about the disease pathogenesis but also provide important targets in development of new generations of medication and treatment.

\section{Materials and methods}

\section{Patients and participants}

A total of 424 individuals from 100 Danish nuclear families selected for clinical atopy were enrolled in the Danish allergy project ITA, The Inheritance of Type I Allergy. The families were recruited from the western part of Denmark through one adult and four paediatric outpatient allergy clinics in Aarhus, Herning, Viborg, and Aalborg. All participants, 200 parents and 224 children, were clinically examined and questionnaire tested by the same doctor. They had blood drawn for DNA analysis and for serum measurement of total IgE and specific IgE, RAST, to 11 common allergens. Among the offspring mean age was 10.8 years and male/female sex ratio was 1.2 equivalent to random distribution $(P=0.35)$. The ITA-project was carried out in accordance with the Helsinki declaration and approved by the Danish scientific ethical committee system. All participants and/or their parents gave informed consent.

\section{Phenotype definition}

To perform the total genome scan, families holding at least two full siblings with clinical allergic rhinitis and elevated specific IgE against at least one of the tested allergens, RAST $\geqslant 1+$, were selected. The use of a narrow phenotype has been discussed earlier. ${ }^{4}$ The specific IgE measurements, CAP RAST FEIA (Pharmacia, Uppsala, Sweden), were elevated when $\geqslant 0.35 \mathrm{kUA} / \mathrm{l}$ ( $\geqslant$ class 1 ) and determined for 11 allergens: grass, birch, mugworth, olive, Parietaria, cat, dog, horse, mite (D. pteronyssinus and D. farinae) and Cladosporium herbarum. Allergic rhinitis was diagnosed according to standard criteria $^{2}$ and determined by two separate doctors - first by one doctor doing all the interviews and examinations of the allergy families in the outpatient clinic and second by one doctor evaluating the questionnaires only. The agreement on the clinical diagnosis was high (>98\%), in the few cases of discrepancies the questionnaires were re-evaluated by the same two physicians and consensus was obtained. A total of 33 ITA sib-pair families qualified for the genome scan on allergic rhinitis on these conditions (Table 1).

\section{Molecular genetics}

A comprehensive set of 446 highly informative microsatellite markers (http://www.marshmed.org/genetics/) scattered with a mean distance of $7.85 \mathrm{~cm}$ (range: $0-16.71 \mathrm{cM}$ ) throughout the 22 autosomal chromosomes and the $\mathrm{X}$ chromosome were selected. The markers were purchased as scan primers (DNA Technology, Aarhus, Denmark). PCR conditions were optimised to make all primers perform in a multiplex PCR with one to six (mean=3.5) primer sets. Products were analysed in 47 panels of four to fourteen primer sets; the separation and genotyping were made on an ABI 310 Genetic Analyzer (Perkin Elmer, Foster City, CA, USA).

\section{Statistical analysis}

The data from the allergic rhinitis affected sib-pair families were analysed by non-parametric multipoint linkage analysis using the maximum-likelihood-score (MLS) approach. ${ }^{5}$ The computer program MAPMAKER/SIBS ${ }^{6}$ was used for calculation of maximum likelihood IDB (identical-by-decent), exclusion LOD scores and information content. To extract the extra information from families with more than two affected siblings we used the MAPMAKER/SIBS program facility to calculate data based on both independent sib-pairs and non-weighted all sib-pairs. Maximum likelihood and exclusion calculations allowed for dominance variance. An increment step setting of five facilitated computing of LOD scores in a total of 2345 positions. Multipoint exclusion maps were obtained for disease susceptibility models with $\lambda_{\mathrm{s}}$ of 1.2 , $1.5,2,3,5$, and 10 .

\section{Results}

We found linkage of allergic rhinitis to the region 4q24-q27 by a LOD score of 2.83 when computing data using the more conservative independent sib-pairs setting. When we repeated the calculations using the all sib-pairs mode the LOD score was 3.26. Another eight regions, 2q12-q33, 3q13, 4p15-q12, 5q13-q15, 6p24-p23, 12p13, 22q13, and Xp21, showed evidence of linkage detected by LOD scores in the range of $1.04-1.63$. The LOD scores rose in the regions $12 \mathrm{p} 13$ and Xp21 to 1.88 and 1.61, respectively, when all sib-pairs calculations were used.

Results of the multipoint linkage analysis through out the genome are shown as plots by chromosome (Figure 1). Specific data from the nine regions with LOD $>1$ are given

Table 1 Clinical features of the allergic rhinitis sib-pair families

\begin{tabular}{lll}
\hline & Parents & Offspring \\
\hline Total number & 66 & 79 \\
Allergic rhinitis & 24 & 73 \\
RAST $\geqslant 1+$ & 28 & 74 \\
Steroid medication & 25 & 65 \\
Males/females & $33 / 33$ & $39 / 40$ \\
Mean age (years) & 44.1 & 14.5 \\
\hline
\end{tabular}

Absolute numbers of family members are listed in six categories. RAST $\geqslant 1+$ indicates specific allergy to at least one of the eleven tested allergens. Steroid medication is local or/and systemic and stated at the time of the clinical examination 
Table 2 Specified results from nine potential candidate regions

\begin{tabular}{|c|c|c|c|c|}
\hline Genetic position & Relative position & Marker (cM) & Info & Lod \\
\hline \multirow[t]{2}{*}{$2 q 12-q 33$} & $139.26(\operatorname{loc} 17)$ & $\mathrm{D} 2 \mathrm{~S} 1241$ & 0.90 & 1.26 \\
\hline & 143.83 (loc 18 ) & D2S442 & 0.91 & 1.47 \\
\hline $3 q 13$ & 120.18 (loc 14$)$ & D3S2460 & 0.84 & 1.63 \\
\hline \multirow{2}{*}{$4 p 15-q 12$} & 47.73 & & 0.90 & 1.35 \\
\hline & 48.49 (loc 8$)$ & D4S3255 & 0.94 & 1.18 \\
\hline $4 q 24-q 27$ & $104.13($ loc 14$)$ & D4S1651 & 0.63 & 1.66 \\
\hline \multirow{3}{*}{$5 q 13-q 15$} & 105.29 (loc 13) & D5S1462 & 0.92 & 1.04 \\
\hline & 108.07 (loc 14$)$ & D5S1503 & 0.89 & 1.10 \\
\hline & 111.85 & & 0.69 & 1.23 \\
\hline \multirow{3}{*}{ 6p24-p23 } & $0.00(\operatorname{loc} 1)$ & D6S277 & 0.64 & 1.50 \\
\hline & 4.84 & & 0.55 & 1.60 \\
\hline & 12.10 (loc 2$)$ & D6S1006 & 0.70 & 1.32 \\
\hline
\end{tabular}

Regions presenting LOD scores $>1$ are listed both by genetic and relative positions. Elevated LOD scores obtained between markers are included to place a potential candidate gene in relation to a close marker. LOD scores $>2$ and relating markers are shown in bold. Information content (Info) is given for all suggestive positions

(Table 2). Information content reflects both marker density and marker heterozygosity and is useful in predicting the quality of the marker map by means of providing exhaustive information in the sample tested. The information content mean value in the nine appointed regions (Table 2) was 0.77 (range $0.44-0.95$ ). Exclusion LOD scores assuming $\lambda_{\mathrm{s}}=3$ and 5 were exclusive (LOD $<-2$ ) for $35 \%$ and $63 \%$ of the genome, respectively. No evidence of linkage was found to either of the atopy candidate genes, TNF- $\beta$ and FcER $1 \beta$, or to the Il4R-region.

\section{Discussion}

Over the past decade intensive research in the complex genetics of allergy has generated numerous reports of linkage and association. The majority of studies have evaluated the phenotypes asthma and atopy, a few have focused on atopic dermatitis, and only one paper has reported data on allergic rhinitis. ${ }^{7}$ The genetic model for allergic disease still remains to be determined, but a combination of general allergy genes and organ specific genes has been suggested. ${ }^{7-9}$

In the present study we evaluated the clinical phenotype allergic rhinitis combined with documented type I allergy, RAST $\geqslant 1+$. The narrow phenotype definition was used in attempt to decrease the genetic heterogeneity and thereby increase the power of the study. ${ }^{4}$ Evidence of linkage between allergic rhinitis and the markers D4S1651 and D4S2394 defined a candidate region of approximate $13 \mathrm{~cm}$ that may harbour a specific susceptibility gene, since the region is novel, also in the general family of potential allergy candidate regions. When observing the allele-sharing pattern in the region the genetic contribution was most consistent with dominant inheritance.

Among the other eight regions showing evidence of linkage two regions were supportive of previous findings in chromosome $2 \mathrm{q} 33^{10}$ and $6 \mathrm{p} 23^{10-14}$ regarding the phenotypes asthma and atopy. We did not find evidence of linkage to an earlier reported allergic rhinitis candidate region $12 \mathrm{q} 14-\mathrm{q} 24,{ }^{15}$ the markers D12S375, D12S1052 and D12S326 (chromosome 12 locus 11-13) all gave LOD scores of 0.00 . Genetic heterogeneity and the mode of sampling may explain this clear divergence between the two studies. In the previous study the families were collected in Barbados and primarily selected for the asthma phenotype. Further more we tested three general atopy candidate genes, the $\operatorname{TNF} \beta$ (chromosome 6 locus 6), the FcER1 $\beta$ (chromosome 11 locus 9) and the Il-4 receptor alpha-subunit region between locus $7-8$ on chromosome 16 , and LOD scores of $0.13,0.06$ and 0.41 , respectively, gave no support for linkage.

Interpretation of data from a genome-scan calls for awareness of increased risk of false-positive results due to multiple testing. A statistical significance level for a genomewide search with a genome-wide false-positive rate $\leqslant 5 \%$ is a LOD score of $4.0(P=0.00002)$ has been recommended when using at dense markermap. ${ }^{16}$ Simulation studies have shown, that if no susceptibility locus is present in the genome, a LOD score $\geqslant 2.3(P \leqslant 0.001)$ will occur by chance somewhere in the genome with a probability of $0.8 .{ }^{6}$ Consequently, LOD scores in the range of 2-3 in new candidate regions must be regarded with some caution, until further evidence has been provided. A closer analysis of this region is ongoing. 
In conclusion, we sampled a thoroughly described clinical set of allergy-sib-pair families and conducted the first genomescan in search for allergic rhinitis candidate genes. Narrow phenotype definition and a comprehensive set of 446 highly informative markers were used. One candidate region of suggestive significance for allergic rhinitis on chromosome

a
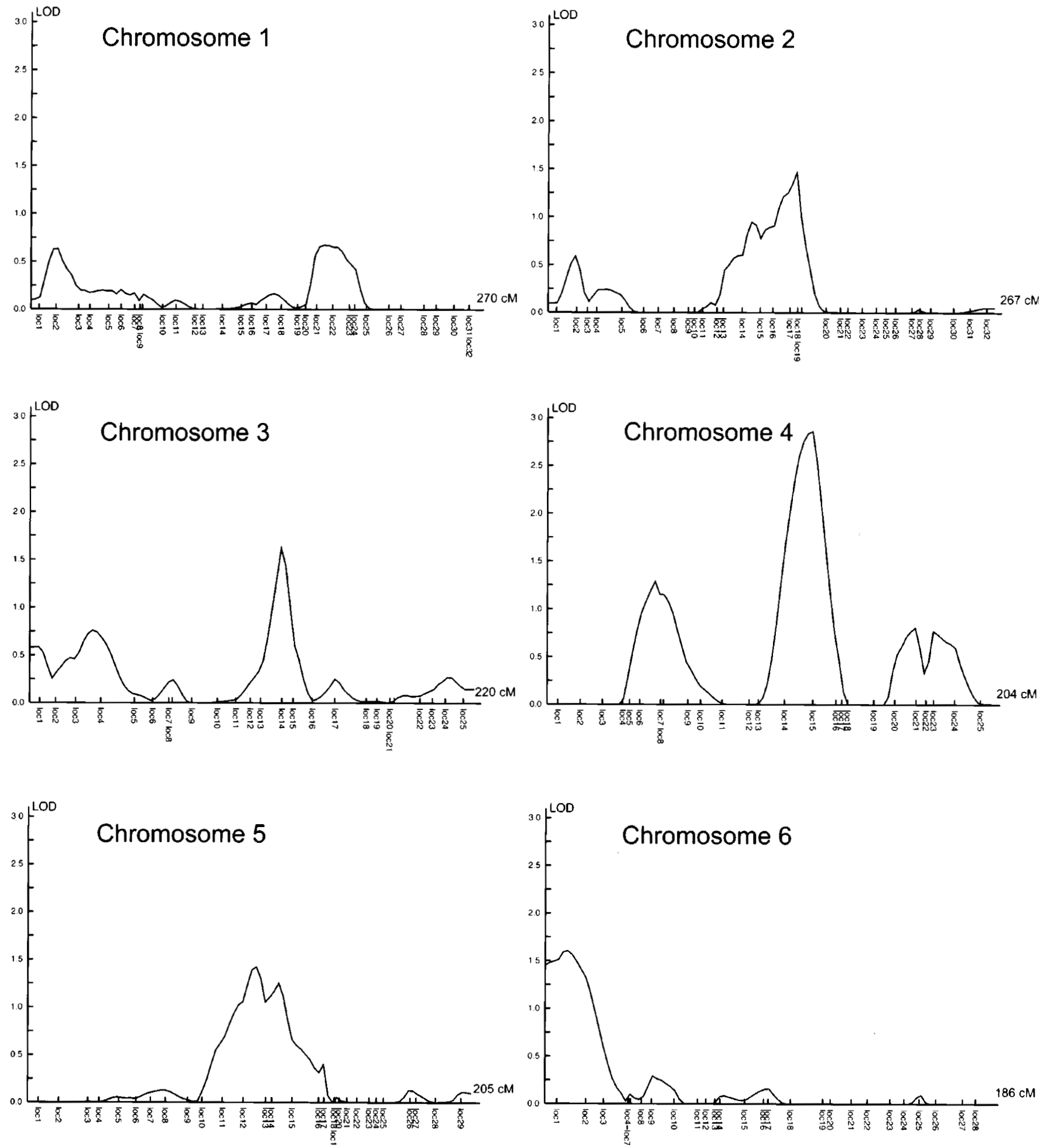
b
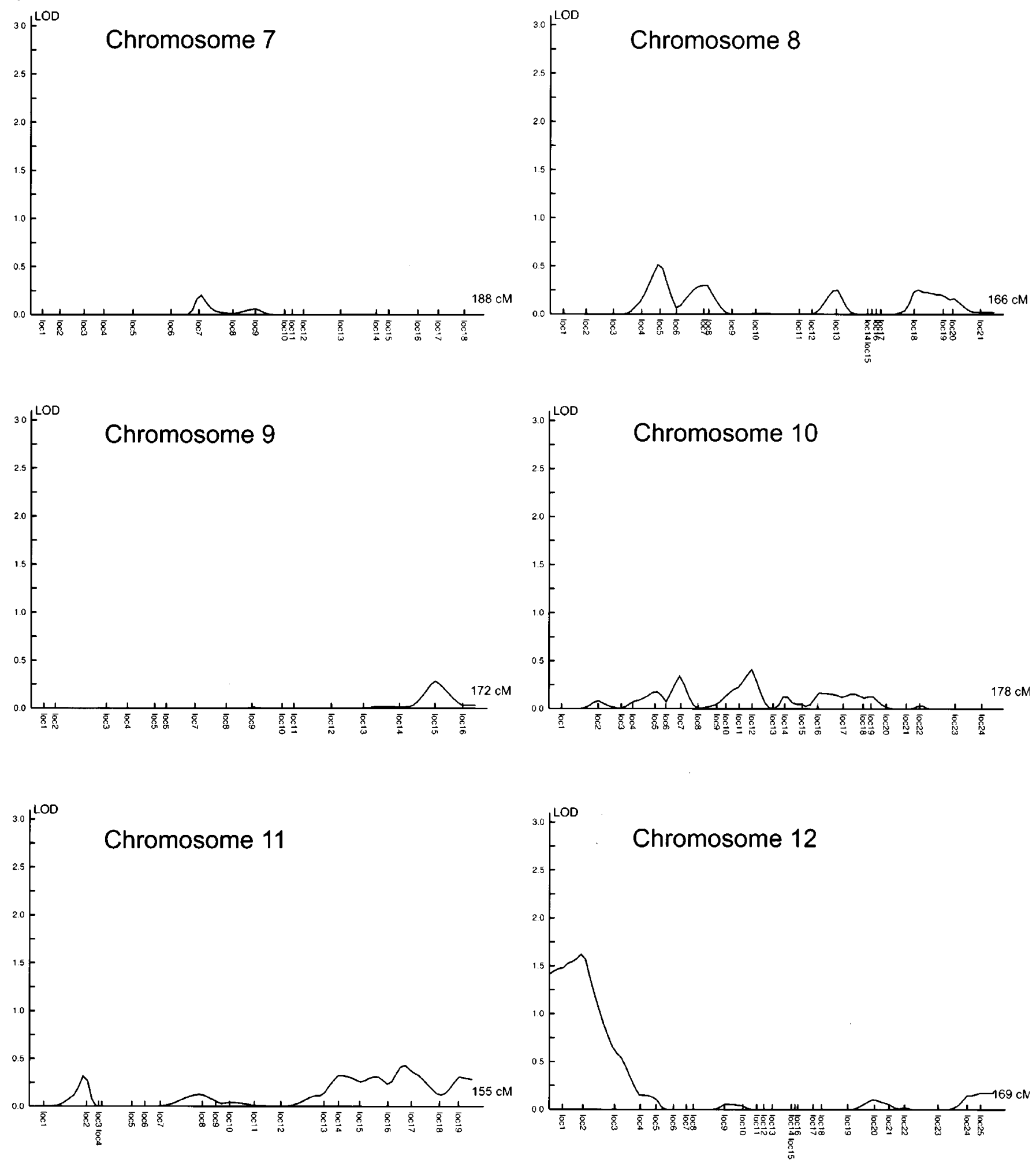

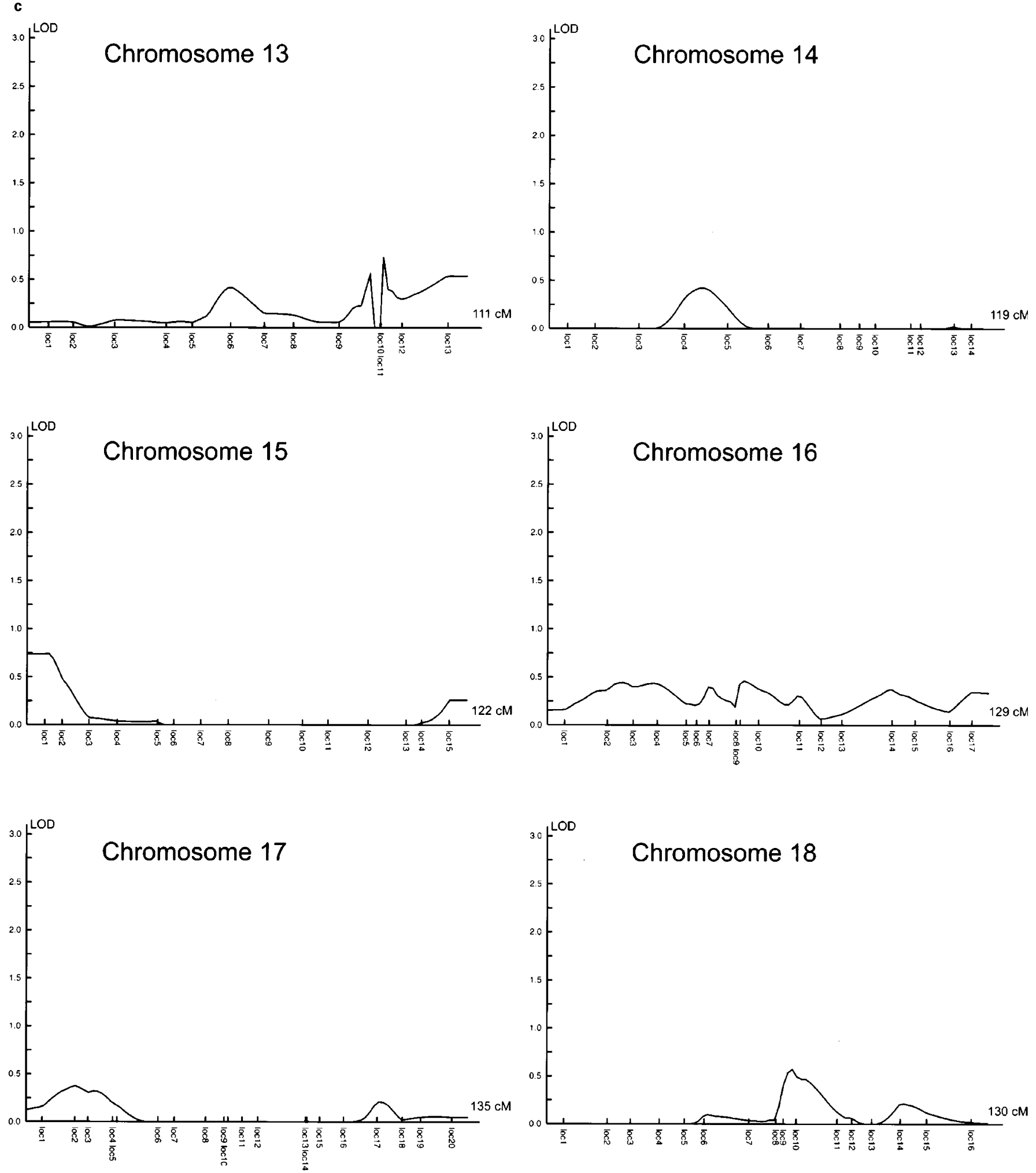
d
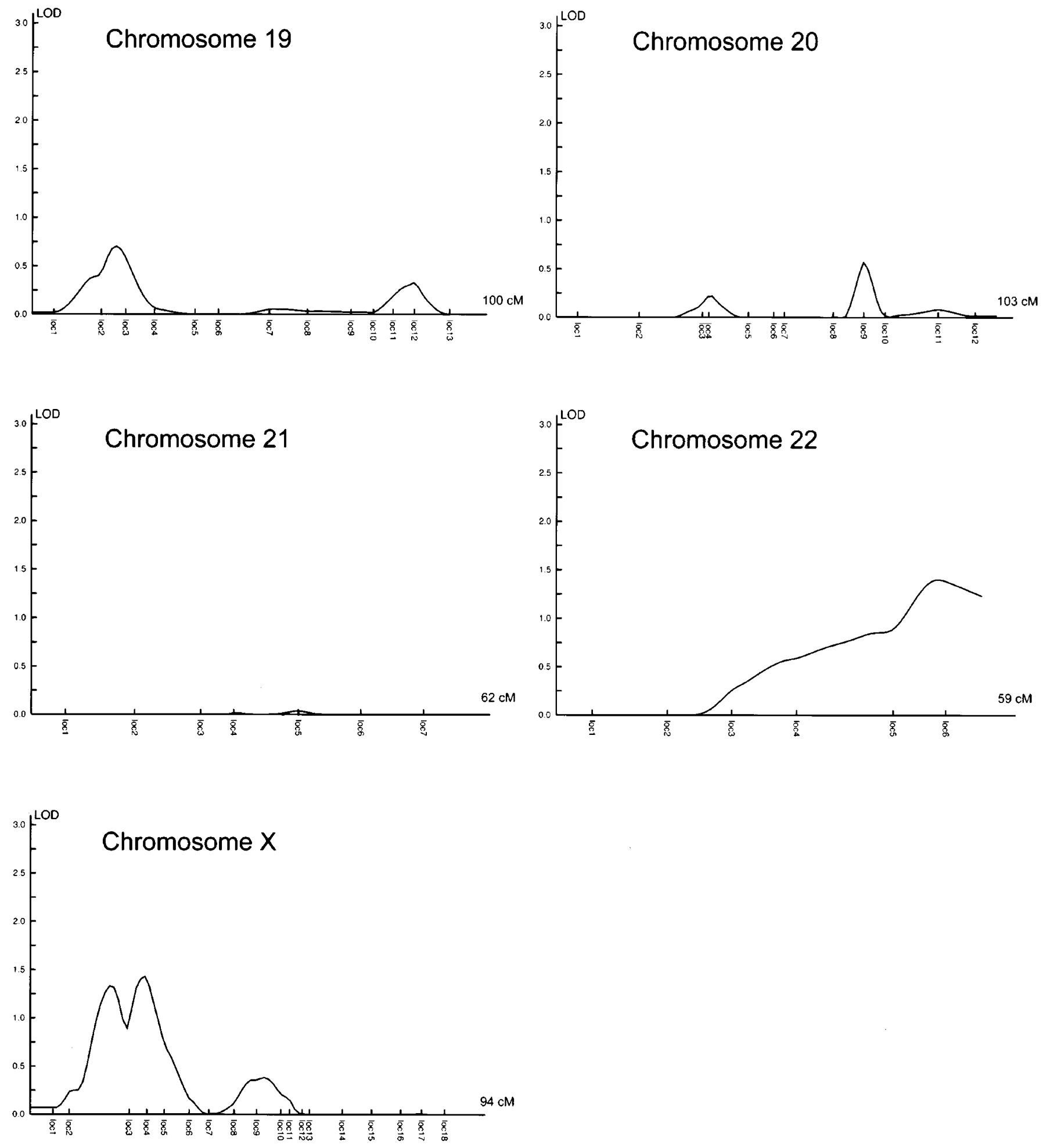

Figure 1 (A-D) Maximum likelihood IBD (MLS) curves for 446 loci (loc) on 23 chromosomes. LOD scores are computed for all markers and five times between every two markers, increment steps of 5 , adding up a total of 2345 locations. 
4q24-q27 was identified by conservative statistical analysis. Moreover, we found evidence supporting an additional eight regions. Our future research will be dedicated fine-scale mapping and investigation of candidate genes in the appointed region.

\section{Acknowledgments}

We thank patients and their families for participating in the ITAproject. The enthusiastic co-operation by the staff of the five Danish outpatient allergy clinics in Aalborg, Viborg, Herning and Aarhus during recruitment of the allergy families is greatly acknowledged. The project has been funded by Ville Heises Fund; the Faculty of Health Sciences, Aarhus University; Hørslev Fund; Danish Lung Association; Danish Allergy Research Centre; The Danish Asthma-Allergy Association, Mimi and Victor Larsen's Fund and ALK Abello Denmark.

\section{References}

1 Holgate S, Church M: Allergy: Mosby-Wolfe, 1995, vol 17, pp 1 10.

2 Dykewicz MS, Fineman S, Skoner DP: Joint Task Force summary statements on Diagnosis and Management of Rhinitis. Ann Allergy Asthma Immunol 1998; 81(5 Pt 2): 474-477.

3 Rasanen M, Laitinen T, Kaprio J, Koskenvuo M, Laitinen LA: Hay fever - a Finnish nationwide study of adolescent twins and their parents. Allergy 1998; 53(9): 885-890.

4 Haagerup A, Bjerke T, Schøitz P, Dahl R, Binderup H, Kruse T: No linkage and association of atopy to chromosome 16 including the interleukin-4 receptor gene. Allergy 2001; 56(8), $775-779$.

5 Risch N: Linkage strategies for genetically complex traits. III. The effect of marker polymorphism on analysis of affected relative pairs [published erratum appears in Am J Hum Genet 1992 Sep; 51(3): 673-675] [see comments]. Am J Hum Genet 1990; 46(2): $242-253$.
6 Kruglyak L, Lander ES: Complete multipoint sib-pair analysis of qualitative and quantitative traits [see comments]. Am J Hum Genet 1995; 57(2): 439-454.

7 Barnes KC: Evidence for common genetic elements in allergic disease. J Allergy Clin Immunol 2000; 106(5 Suppl): S192 - S200.

8 Haagerup A, Bjerke T, Schiøtz P, Dahl R, Binderup H, Kruse T: Type I allergy - a total genomescanning for candidate region and test of candidate genes. Am J Hum Genet 1999; 65(4): A272.

9 Forrest S, Dunn K, Elliott K et al: Identifying genes predisposing to atopic eczema. J Allergy Clin Immunol 1999; 104(5): 10661070.

10 CSGA: A genome-wide search for asthma susceptibility loci in ethnically diverse populations. The Collaborative Study on the Genetics of Asthma (CSGA). Nat Genet 1997; 15(4): 389-392.

11 Caraballo LR, Hernandez M: HLA haplotype segregation in families with allergic asthma. Tissue Antigens 1990; 35(4): 182 186.

12 Daniels SE, Bhattacharrya S, James A et al: A genome-wide search for quantitative trait loci underlying asthma. Nature 1996; 383(6597): $247-250$

13 Hizawa N, Freidhoff LR, Chiu YF et al: Genetic regulation of Dermatophagoides pteronyssinus-specific IgE responsiveness: a genome-wide multipoint linkage analysis in families recruited through 2 asthmatic sibs. Collaborative Study on the Genetics of Asthma (CSGA). J Allergy Clin Immunol 1998; 102(3): 436442.

14 Wjst M, Fischer G, Immervoll T et al: A genome-wide search for linkage to asthma. German Asthma Genetics Group. Genomics 1999; 58(1): $1-8$.

15 Barnes KC, Freidhoff LR, Nickel R et al: Dense mapping of chromosome 12q13.12-q23.3 and linkage to asthma and atopy. I Allergy Clin Immunol 1999; 104(2 Pt 1): $485-491$.

16 Lander ES, Schork NJ: Genetic dissection of complex traits [published erratum appears in Science 1994 Oct 21; 266(5184): 353]. Science 1994; 265(5181): $2037-2048$. 